\title{
DELAYED RESUSCITATION WITH PHYSOSTIGMINE INCREASES END ORGAN DAMAGE IN ALCOHOL INTOXICATED RATS
}

\author{
Jesse K. Sulzer and Patricia E. Molina \\ Louisiana State University Health Sciences CenterDepartment of Physiology and Alcohol and \\ Drug Abuse Center of Excellence, New Orleans, LA 70112
}

\begin{abstract}
Previous studies from our laboratory have identified a role for blunted central sympathetic activation in the acute alcohol intoxication (AAI)-induced impairment of the counterregulatory response to hemorrhagic shock (HS). Immediate fluid resuscitation (FR) with acetylcholinesterase-inhibitors restores the neuroendocrine and pressor responses to FR in AAI + HS. We hypothesized this intervention would remain beneficial following delay and that restoration of MABP during FR would attenuate organ damage. Male Sprague-Dawley rats received a primed constant alcohol infusion $(2.5 \mathrm{~g} / \mathrm{kg}+0.3 \mathrm{~g} / \mathrm{kg} / \mathrm{h}$ for $15 \mathrm{~h})$ or isocaloric dextrose (DEX) prior to HS (40 mmHg for $60 \mathrm{~min}$ ) and FR with lactated Ringer's (LR) \pm physostigmine (PHYS; $100 \mathrm{ug} / \mathrm{kg}$ ) immediately or following a $60 \mathrm{~min}$ delay post-HS. Immediate LR elevated MABP in DEX+HS. AAI delayed the initial MABP recovery. Delayed LR did not further increase MABP in DEX- or AAI+HS. LR+PHYS increased MABP in DEX- and AAI+HS following immediate and delayed FR. No differences were noted in markers of organ dysfunction (ALT, AST, BUN, creatinine) following DEX+HS and this was unaltered by immediate or delayed LR +PHYS. AAI+HS increased ALT, which was attenuated by immediate LR+PHYS. In contrast, delayed LR+PHYS exacerbated tissue injury in AAI+HS, as reflected by increased ALT, AST, BUN, creatinine, and liver protein carbonylation over time-matched LR. In conclusion, PHYS enhanced blood pressure recovery independent of time of FR and presence of AAI. However, in AAI+HS, delayed LR+PHYS accentuated organ damage and dysfunction. These findings suggest that while enhancing the sympathetic response can improve hemodynamic recovery during AAI, it may compromise tissue perfusion and enhance tissue injury.
\end{abstract}

\section{Keywords}

alcohol intoxication; acetylcholinesterase inhibitors; hemorrhage; organ injury; reperfusion; vasopressors

\section{INTRODUCTION}

Acute alcohol intoxication (AAI) is a common risk factor for traumatic injury that complicates the initial management of trauma victims (1-3). Intoxication at the time of injury contributes to a greater hypotension at the time of arrival to the emergency department and increased injury severity scores (4). Studies from our laboratory demonstrate that AAI leads to a dysregulation of the counterregulatory response to hemorrhagic shock (HS) including an impaired hemodynamic response to blood loss and fluid resuscitation that is associated with a blunted neuroendocrine response. AAI blunts the hemorrhage-induced

Address correspondence to: Patricia E. Molina, MD, PhD, LSUHSC Department of Physiology, 1901 Perdido Street, New Orleans, LA 70112-1393, Phone: 504-568-6171, Fax: 504-568-6158, pmolin@1suhsc.edu. 
increase in circulating levels of the counterregulatory hormones and potent vasopressors epinephrine, norepinephrine, and arginine vasopressin $(5,6)$. Investigations into the mechanism of this impaired counterregulatory response have ruled out impaired vascular pressor response as a primary mechanism for the greater hypotension following blood loss observed in the intoxicated host (7). In contrast, studies examining the role of the blunted sympathetic response to hemorrhage have provided strong support for the hypothesis that the impaired response to blood loss during AAI is due to central alterations in sympathetic outflow $(8,9)$. Central administration of acetylcholinesterase inhibitors increases sympathetic outflow, significantly improves the blunted counterregulatory hormone response associated with AAI, and contributes to an enhanced blood pressure recovery following hemorrhage (8). Further studies investigating the therapeutic potential of this approach have confirmed the ability of peripherally administered acetylcholinesterase inhibitors, such as physostigmine, to restore the neuroendocrine response and improve the pressor response to fluid resuscitation following hemorrhagic shock during AAI (10).

In examining the potential clinical benefit to utilization of acetylcholinesterase inhibitors in the intoxicated trauma victim, additional factors must be considered. Conventional approaches dictate that trauma patients receive definitive care within the first "golden hour" (11). Modern trauma systems are largely based on this principle of rapid care (12). However, treatment can often be delayed from the time of injury, such as during a prolonged entrapment. This delay in resuscitation has a significant impact on patient outcome $(13,14)$. The current study sought to investigate the ability of the acetylcholinesterase inhibitor physostigmine to contribute to improved blood pressure recovery when administered after a delay. In addition, we examined whether improved restoration of MABP would confer protection from organ damage in alcohol-intoxicated hemorrhaged rats. We hypothesized that physostigmine would retain the ability to enhance blood pressure recovery following delayed treatment. Additionally, we predicted that the improved hemodynamic recovery achieved with physostigmine resuscitation would be associated with decreased organ damage and dysfunction.

\section{MATERIALS AND METHODS}

\section{Animals}

Specific pathogen-free adult male Sprague-Dawley rats (250-275g; Charles River, Raleigh, $\mathrm{NC})$ were housed in a controlled-temperature $\left(22^{\circ} \mathrm{C}\right)$ and controlled-illumination (12-h light/ dark cycle) environment for one week prior to surgical procedures during which they were allowed standard rat chow (Purina, St. Louis, MO) and water ad libitum. All animal procedures were approved by the Institutional Animal Care and Use Committee at Louisiana State University Health Sciences Center and were performed in accordance with the guidelines of the National Institutes of Health.

\section{Surgical Procedures}

Animals were anesthetized with ketamine/xylazine ( $90 \mathrm{mg} / \mathrm{kg}$ and $9 \mathrm{mg} / \mathrm{kg}$ respectively) and using aseptic surgical technique, sterile catheters (PE50) were placed into the carotid artery and jugular vein for blood pressure monitoring, blood sample collection, and drug and fluid resuscitation administration (15). An additional catheter was placed into the antrum of the stomach for alcohol administration. All catheters were routed subcutaneously and exteriorized through a small incision in the nape of the neck. At the completion of all surgical procedures, the catheters were secured with tape and animals returned to their cages for 2-3 days to recover, during which they were given free access to food and water. 


\section{Alcohol Administration}

On the day prior to hemorrhage, animals received an intragastric bolus of $2.5 \mathrm{~g} / \mathrm{kg}$ of $30 \%$ ethanol followed by a 15 -hour constant infusion at a rate of $300 \mathrm{mg} / \mathrm{kg} / \mathrm{h}$. This protocol provides a total of approximately $7 \mathrm{~g} / \mathrm{kg}$ and produced blood alcohol levels of $223 \pm 9.8 \mathrm{mg} /$ dl. This mimics an episode of binge drinking, defined as consumption of five or more drinks over a period of time sufficient to elevate blood alcohol content above intoxicating levels (16). Binge drinking has been shown to increase the risk of traumatic injury and other alcohol-related deaths in otherwise healthy persons (17-18) Control animals received an equal volume of isocaloric dextrose (52\%) solution $(12.2 \mathrm{~g} / \mathrm{kg})$.

\section{Fixed-Pressure Hemorrhagic Shock}

Following the completion of alcohol administration, conscious and unrestrained animals were bled from the carotid catheter to achieve a mean arterial pressure of $40 \mathrm{mmHg}$. Hypotension was maintained between 40 and $55 \mathrm{mmHg}$ by removal of additional blood as necessary. MABP was continuously monitored during the hemorrhage period (Powerlab, AD Instruments, Colorado Springs, CO). Blood samples were collected at the onset of hemorrhage for blood alcohol levels.

\section{Fluid Resuscitation}

Two studies were conducted to compare the relative benefit of physostigmine administration immediately following the hemorrhage period or after a 60 minute delay following completion of the bleedout period. In the first study, animals were resuscitated immediately following completion of the 60 minute hemorrhage period. In the second study, animals were subjected to a one hour delay prior to the onset of resuscitation (60 minute hemorrhage + 60 minute hypotensive delay). No additional blood was removed nor any fluids administered during the 60 minute delay. Fluid resuscitation consisted of lactated Ringer's administered as a bolus equal to $40 \%$ of the total blood removed followed by a constant infusion of $20 \mathrm{ml}$ over 60 minutes $(1.8 \times$ total blood removed for AAI and $1.7 \times$ total blood removed for dextrose-treated). This resuscitation protocol was used to restore blood pressure to similar values in both alcohol-intoxicated and control animals. Previous studies utilizing a fluid resuscitation model based on total blood removed have demonstrated that AAI impairs the response to fluid resuscitation $(5,6)$. Adapting the volume of fluid resuscitation in the AAI-hemorrhage animals produces a similar blood pressure recovery in both groups and allowed for isolation of the effects of physostigmine administration. This model reflects how physostigmine could be applied with current clinical resuscitation protocols defined by Advanced Trauma Life Support in which hypotensive patients are initially resuscitated with a standard bolus of 2 liters of lactated Ringer's. Animals were randomly selected to receive an intravenous bolus of the acetylcholinesterase inhibitor physostigmine $(100 \mu \mathrm{g} / \mathrm{kg} ; \sim 300$ $\mu \mathrm{L} ;$ Sigma, St. Louis, MO) or an equal volume of vehicle (300 $\mu \mathrm{L}$ normal saline) as an adjuvant at the onset of fluid resuscitation. This dose of physostigmine was based on previous studies from our laboratory that established the minimal dose of physostigmine that had maximal effectiveness in restoring blood pressure following removal of $40 \%$ of total blood volume (10). MABP was continuously monitored during the resuscitation period. Arterial blood samples $(1.5 \mathrm{ml})$ were collected at the end of resuscitation for determination of circulating makers of tissue injury and replaced with an equal volume of normal saline. At completion of fluid resuscitation, venous, arterial, and gastric catheters were secured, and animals were allowed to recover with access to water and food ad libitum in a controlled (temperature and light cycle) environment for up to 7 days post-hemorrhagic shock. 


\section{Blood Sample Analysis}

Blood samples were collected in chilled heparinized syringes, placed in tubes containing 10 $\mu \mathrm{l} / \mathrm{ml}$ aprotinin (Sigma, St. Louis, MO), and centrifuged for 10 minutes at 10,000 RPM for plasma separation. Plasma alcohol levels were measured using an amperometric oxygen electrode and the respective kit (Analox Instruments Limited, London, England). Alanine aminotransferase (ALT) and aspartate aminotransferase (AST) values were determined as makers of liver damage using colorimetric reagent kits based on the oxidation of NADH (Stanbio, Boerne, Tx). Blood urea nitrogen (BUN) and creatinine were determined as indices of renal dysfunction and damage using QuantiCrom assay kits (BioAssay Systems, Hayward, CA).

\section{Liver Protein Carbonylation}

Levels of protein carbonyls were utilized as a measure of oxidative damage in liver tissue at 7 days post-hemorrhage. The liver, as the principle organ of alcohol metabolism, is particularly vulnerable following the combined insult of AAI and hemorrhage. Protein carbonylation was determined by a commercially available reagent kit (Cayman Chemical, Ann Arbor, MI) utilizing the method of Levine et al (19). Briefly, 200 mg tissue was homogenized in $50 \mathrm{mM}$ phosphate buffer and an aliquot was labeled with 2, 4dinitrophenylhydrazine (DNPH). Formed derivatives were extracted with $20 \%$ trichloroacetic acid, centrifuged at 10,000 RPM at $4^{\circ} \mathrm{C}$ for 10 minutes and the pellet resuspended in 10\% trichloroacetic acid followed by 3 treatments of 1:1 ethanol-ethylacetate prior to resuspension in guanidine hydrochloride with centrifugation at 10,000 RPM for 10 minutes at $4^{\circ} \mathrm{C}$ between each treatment. Absorbance was then determined at $370 \mathrm{~nm}$ on a plate reader (BioRad; Hercules, CA) and adjusted for the absorbance of $2.5 \mathrm{M} \mathrm{HCl}$ control samples. Results are presented as nmol of protein carbonyls per $\mathrm{mg}$ total protein as determined by a BCA Protein Assay Kit (Thermo Scientific, Rockford, IL).

\section{Statistical Analysis}

All data are presented as mean \pm standard error of the mean (SEM) with the number of animals used per group indicated in the figure legends. Statistical analysis was accomplished using one- or two-way analysis of variance (ANOVA) with or without repeated measures (Sigma Stat; San Jose, CA) as indicated in each figure legend. Post-hoc pair-wise multiple comparisons were completed with the Holm-Sidak method. Statistical significance was set at $\mathrm{p}<0.05$.

\section{RESULTS}

\section{Hemodynamic response to hemorrhage}

Alcohol-intoxicated animals had lower basal mean arterial blood pressure values than those of dextrose- treated controls (112 vs. $120 \mathrm{mmgHg}$; $\mathrm{p}<0.05$ ). MABP during the hemorrhage period was similar in all animals under all experimental conditions (figure 1). Alcohol intoxication decreased the total blood volume removed necessary to produce and maintain a mean pressure of $40 \mathrm{mmHg}$ ( $57 \mathrm{vs.} 64 \%$; $\mathrm{p}<0.001$ ). Both alcohol intoxicated and dextrosetreated hemorrhaged animals remained hypotensive during the 60 minute delay period before initiation of fluid resuscitation in the delayed treatment group (figure 1).

\section{Hemodynamic response to resuscitation}

Immediate fluid resuscitation following the hemorrhage period with LR alone produced a significant increase in mean arterial blood pressure (figure 2). This increase was significantly greater in response to the initial bolus in dextrose-hemorrhage animals (97\% increase) than in the alcohol-hemorrhage (27\% increase). Physostigmine administration 
enhanced blood pressure recovery compared to LR alone in alcohol (21\%) and dextrosehemorrhage animals (21\%) when administered immediately following hemorrhage.

During the 60 min delay between completion of the hemorrhage protocol and initiation of fluid resuscitation in the delayed treatment group, there was a similar rise in blood pressure in alcohol- $(29 \pm 7 \mathrm{mmHg}$ increase) and dextrose-treated $(28 \pm 6 \mathrm{mmHg}$ increase) animals to $73 \pm 3$ and $80 \pm 5 \mathrm{mmHg}$ respectively. Following the $60 \mathrm{~min}$ delay, fluid resuscitation with LR alone failed to further improve blood pressure in alcohol and dextrose hemorrhage animals (figure 2). Delayed fluid resuscitation with LR and physostigmine produced a significant increase in mean arterial blood pressure in both dextrose (30\%) and alcohol-intoxicated hemorrhaged animals (29\%). Mean arterial blood pressure was significantly higher at completion of resuscitation when combined with physostigmine than LR alone in both dextrose and alcohol-intoxicated hemorrhage animals.

\section{Organ injury and dysfunction}

ALT and AST-Circulating levels of ALT were not different following hemorrhage in dextrose-treated animals resuscitated with LR, nor were levels affected by immediate resuscitation with physostigmine (figure 3). Acute alcohol intoxicated hemorrhaged animals showed a significant increase in ALT ( $\sim .6$ fold vs. time-matched alcohol-treated shams). Immediate physostigmine administration attenuated the hemorrhage-induced rise in ALT in intoxicated animals. Delayed resuscitation with LR did not result in further hemorrhageinduced increases in the circulating levels of ALT in dextrose- or alcohol-treated animals. However, when physostigmine was added to delayed fluid resuscitation, a significant increase in ALT levels ( $~ 9.8$ fold vs. time-matched shams; 2.6 -fold vs. time- and treatmentmatched LR-treated animals) was observed.

AST levels were increased at completion of resuscitation in both alcohol- and dextrosetreated animals and this was not altered by immediate resuscitation with physostigmine (figure 3). Delayed resuscitation in dextrose-hemorrhage animals resulted in a similar rise in levels of AST in animals receiving LR, which was attenuated by physostigmine administration. In contrast, delayed resuscitation with physostigmine further enhanced the hemorrhage-induced rise in AST ( 2.5 fold vs. time- and treatment-matched LR-treated animals) in alcohol-intoxicated animals.

BUN and Creatinine-Resuscitation with LR alone did not alter plasma creatinine levels in alcohol- or dextrose-treated animals (figure 4). Immediate physostigmine administration increased circulating levels of creatinine (54\% increase over time-matched sham) in dextrose but not in the alcohol-intoxicated hemorrhaged animals. Delayed resuscitation with LR resulted in a further increase in creatinine in dextrose but not alcohol-intoxicated hemorrhaged animals. Delayed resuscitation with physostigmine did not alter the hemorrhage-induced rise in creatinine in dextrose-treated, but accentuated the increase in circulating levels of creatinine in alcohol-intoxicated hemorrhaged animals (44\% increase vs. time- and treatment-matched LR-treated animals).

BUN levels were increased following hemorrhage in both alcohol and dextrose-treated animals, and this increase was attenuated with immediate resuscitation with physostigmine (figure 4). Delayed resuscitation with or without physostigmine resulted in no further increases in BUN in dextrose-hemorrhage animals. In contrast, delayed resuscitation with physostigmine in alcohol-intoxicated hemorrhaged animals resulted in a significant increase in circulating levels of BUN over those of time- and treatment-matched LR-treated animals.

Liver protein carbonylation-Protein carbonylation was not altered by hemorrhage alone in either alcohol or dextrose animals that received immediate or delayed fluid 
resuscitation with LR alone (figure 5). Immediate or delayed administration of physostigmine did not alter the levels of protein carbonylation in dextrose-hemorrhage animals. However, delayed resuscitation with physostigmine led to significantly higher levels of protein carbonyls in liver tissue of alcohol-intoxicated animals.

\section{DISCUSSION}

Physostigmine administration at the time of fluid resuscitation from hemorrhagic shock has previously been demonstrated to restore the neuroendocrine and hemodynamic responses in alcohol-intoxicated rodents. In the present study, we examined whether delayed administration would be similarly effective and, furthermore, whether it would improve hemodynamic recovery without untoward effects. The results from this study confirmed the benefits of systemic acetylcholinesterase inhibitor administration on hemodynamic recovery from hemorrhage in the alcohol-intoxicated host. Moreover, they demonstrate continued benefit in blood pressure recovery even after substantial delay in administration irrespective of alcohol intoxication. However, while immediate resuscitation with physostigmine was associated with attenuated liver damage in alcohol intoxicated hemorrhaged animals, delayed administration increased multiple indices of organ damage and dysfunction.

The utility of acetylcholinesterase inhibitors as resuscitation tools has been previously demonstrated in non-intoxicated animals. Administration results in benefits including improved blood pressure recovery, increased circulating blood volume, and decreased mortality $(20,21)$. These findings have been expanded in our recent studies, which have demonstrated that the improved hemodynamic recovery following hemorrhagic shock is not diminished by alcohol intoxication $(8,10)$. The impact of this approach on end-organ damage has not been studied extensively. We predicted that the improved blood pressure recovery achieved with this intervention would decrease indices of organ damage following hemorrhage. Physostigmine results in the induction of a significant increase in circulating levels of the potent vasopressors epinephrine, norepinephrine, and arginine vasopressin (10). Exogenous pressor use has been demonstrated to enhance vital organ blood flow and urine output in normotensive animals (22). Pressor use has also been demonstrated to improve outcome during models of endotoxic shock irrespective of time of administration (23). Unfortunately, extrapolation of these interventions to other models of shock have produced varied responses including impaired tissue perfusion and function during resuscitation from hemorrhage (24). Therefore, despite the improvement in mean arterial blood pressure obtained with physostigmine administration, a robust increase in endogenous pressors could have led to decreases in regional or organ-specific blood flow contributing to enhanced organ dysfunction and tissue injury, particularly in the alcohol-intoxicated hemorrhaged animals. This was most evident in animals subjected to the delayed resuscitation protocol and consequently exposed to a prolonged hypotensive period. The prolonged hypotension coupled with the increase in endogenous vasopressors could have produced a decrease in renal perfusion and glomerular filtration leading to the increased BUN and creatinine, markers of renal function, observed with delayed physostigmine administration. While an alcohol effect was noted in makers of renal dysfunction, it was significantly less pronounced than that observed in hepatic markers.

The patient population of interest in this study may be particularly vulnerable to liver damage following hemorrhage and resuscitation (25). Studies have demonstrated that a large proportion of acutely intoxicated trauma victims may additionally be chronic alcohol abusers (26). Therefore, in addition to the additional potential damage resulting from acute alcohol intoxication, these patients may present with preexisting hepatic conditions or injury that predispose them to organ damage and failure $(25,27)$. Animal studies investigating this 
effect have confirmed clinical findings and begun to establish the complex mechanisms of alcohol-induced liver injury following hypotension (28).

A potential mechanism of the observed organ injury in this study is damage by reactive oxygen species resulting from both hepatic alcohol metabolism and the low flow-reperfusion resulting from hemorrhage and fluid resuscitation. This was reflected in the increased liver protein carbonyl content, particularly following delayed fluid resuscitation with physostigmine in alcohol-intoxicated animals. Protein carbonylation is an irreversible marker of oxidative injury resulting from the interaction of reactive oxygen and nitrogen species with side-chain amines (19). Given the period of observation in this study, protein carbonylation provided a stable indicator of previous and cumulative oxidative damage. Hemorrhage has been demonstrated to increase tissue levels of reactive oxygen species and lipid peroxidation products (29). Alcohol intoxication has also been demonstrated to increase free radical production, particularly in the liver (30). Moreover, studies indicate that $\alpha_{1 \mathrm{~B}}$-adrenoceptor activation produces additive effects with ethanol on free radical production in hepatocytes (31). Together, the hemorrhagic shock, alcohol metabolism and excessive adrenergic activation following physostigmine administration could be possible mechanisms underlying the enhanced hepatic injury seen in the intoxicated animals resuscitated with physostigmine following delay. Therefore, despite evidence that oxidative damage can be attenuated by modulation of resuscitation techniques, the specific interactions with alcohol likely enhanced injury in this study and suggest that alternative strategies that improve hemodynamic recovery with limited sympathetic activation may be better suited to this population (29). In particular, studies utilizing alternative fluid resuscitation strategies, such as hypertonic saline, have demonstrated an ability to improve hemodynamic recovery and survival and limit additional negative outcomes and thus may serve as an additional targeted and more physiologic approach for intoxicated trauma victims (32).

The key findings of this study indicate that resuscitation with acetylcholinesterase inhibitors can improve blood pressure recovery following hemorrhage irrespective of time of administration in alcohol-intoxicated and dextrose-treated control animals. Immediate resuscitation with physostigmine was associated with attenuation of the hemorrhage-induced rise in ALT in alcohol-intoxicated animals. In contrast, delayed resuscitation with physostigmine contributed to enhanced hemorrhage-induced organ damage in intoxicated animals. As multiple organ failure remains a common cause of morbidity and mortality in trauma victims, the increased organ damage reported here has significant implications for recovery following injury and blood loss. The altered organ function observed with delayed physostigmine resuscitation in alcohol-intoxicated animals could contribute to enhanced susceptibility to a 'second hit' challenge during the recovery period. The results from this and our recent studies strongly suggest that oxidative damage resulting from the combination of hepatic alcohol metabolism and reperfusion following prolonged hypotension was further aggravated by excess adrenergic stimulation resulting from physostigmine administration. We predict that careful modulation of the sympathetic response will be required to maximize positive outcomes following resuscitation in the alcohol intoxicated trauma victim. While previous work has demonstrated a role for the blunted sympathetic response in the impaired hemodynamic recovery during AAI, clinical studies indicate a strong association between elevated catecholamine levels and mortality (33). In attempting to achieve this delicate balance, more physiologic approaches warrant further investigation. 


\section{Acknowledgments}

The authors would like to thank Christopher Hakenjos, Annie Whitaker, Edith Walker, and Keisa Mathis for their assistance in completing these studies and editorial suggestions.

These studies were supported by DOD-PR-054196, the Louisiana Board of Regents, and NIAAA-AA-7577

\section{References}

1. Hingson R, Heeren T, Winter M, Wechsler H. Magnitude of alcohol-related mortality and morbidity among U.S. college students ages 18-24: changes from 1998 to 2001. Annu Rev Public Health. 2005; 26:259-79. [PubMed: 15760289]

2. Jurkovich GJ, Rivara FP, Gurney JG, Fligner C, Ries R, Mueller BA, Copass M. The effect of acute alcohol intoxication and chronic alcohol abuse on outcome from trauma. JAMA. 1993; 270(1): 51Y56. [PubMed: 8510296]

3. Jurkovich GJ, Rivara FP, Gurney JG, Seguin D, Fligner CL, Copass M. Effects of alcohol intoxication on the initial assessment of trauma patients. Ann Emerg Med. 1992; 21:704-708. [PubMed: 1590611]

4. Shih HC, Hu SC, Yang CC, Ko TJ, Wu JK, Lee CH. Alcohol intoxication increases morbidity in drivers involved in motor vehicle accidents. Am J Emerg Med. 2003; 21(2):91-4. [PubMed: 12671806]

5. Molina PE, Zambell KL, Norenberg K, Eason J, Phelan H, Zhang P, Stouwe CV, Carnal JW, Porreta C. Consequences of alcohol-induced early dysregulation of responses to trauma/ hemorrhage. Alcohol. 2004; 33(3):217-27. [PubMed: 15596090]

6. Mathis KW, Zambell K, Olubadewo JO, Molina PE. Altered hemodynamic counter-regulation to hemorrhage by acute moderate alcohol intoxication. Shock. 2006; 26(1):55-61. [PubMed: 16783199]

7. Molina MF, Whitaker A, Molina PE, McDonough KH. Alcohol does not Modulate the Augmented Acetylcholine-induced Vasodilatory Response in Hemorrhaged Rodents. Shock. 2009; 32(6):601-7. [PubMed: 19197228]

8. Mathis KW, Molina PE. Central acetylcholinesterase inhibition improves hemodynamic counterregulation to severe blood loss in alcohol-intoxicated rats. Am J Physiol Regul Integr Comp Physiol. 2009; 297(2):R437-45. [PubMed: 19515985]

9. Mathis KW, Molina PE. Transient central cholinergic activation enhances sympathetic nervous system activity but does not improve hemorrhage-induced hypotension in alcohol-intoxicated rodents. Shock. 2009; 32(4):410-5. [PubMed: 19197225]

10. Mathis KW, Sulzer JK, Molina PE. Systemic administration of a centrally-acting acetylchoinlesterase inhibitor improves outcome from hemorrhagic shock during acute alcohol intoxication. Shock. Dec 17.2009 [Epub ahead of print].

11. Lerner EB, Moscati RM. The golden hour: scientific fact or medical "urban legend"? Acad Emerg Med. 2001; 8(7):758-60. [PubMed: 11435197]

12. Rady MY. Triage and resuscitation of critically ill patients in the emergency department: current concepts and practice. Eur J Emerg Med. 1994; 1(4):175-89. [PubMed: 9422164]

13. Garcia A. Critical care issues in the early management of severe trauma. Surg Clin North Am. 2006; 86(6):1359-87. [PubMed: 17116453]

14. Cocchi MN, Kimlin E, Walsh M, Donnino MW. Identification and resuscitation of the trauma patient in shock. Emerg Med Clin North Am. 2007; 25(3):623-42. vii. [PubMed: 17826209]

15. Phelan H, Stahls P, Hunt J, Bagby GJ, Molina PE. Impact of alcohol intoxication on hemodynamic, metabolic, and cytokine responses to hemorrhagic shock. J Trauma. 2002; 52(4): 675-82. [PubMed: 11956381]

16. Naimi TS, Brewer RD, Mokdad A, Denny C, Serdula MK, Marks JS. Binge drinking among US adults. Journal of the American Medical Association. 2003; 289(1):70-75. [PubMed: 12503979]

17. Savola O. Alcohol intake and the pattern of trauma in young adults and working aged people admitted after trauma. Alcohol Alcohol. 2005; 40(4):269-273. [PubMed: 15870091] 
18. Gmel G, Bissery A, Gammeter R, Givel JC, Calmes JM, Yersin B, Daeppen JB. Alcoholattributable injuries in admissions to a Swiss emergency room--an analysis of the link between volume of drinking, drinking patterns, and preattendance drinking. Alcoholism: Clinical and Experimental Research. 2006; 30(3):501-509.

19. Levine RL, Garland D, Oliver CN, Amici A, Climent I, Lenz AG. Determination of carbonyl content in oxidatively modified proteins. Meth Enzymol. 1990; 186:464-78. [PubMed: 1978225]

20. Guarini S, Tagliavini S, Ferrari W, Bertolini A. Reversal of haemorrhagic shock in rats by cholinomimetic drugs. British Journal of Pharmacology. 1989; 98(1):218-24. [PubMed: 2804546]

21. Savic J, Vargic VM, Prokic DJ, Vujnov S, Prostran M, Zunic G, Stanimirovic D, RastovacBogdanovic M. The life-saving effect of physostigmine in haemorrhagic shock. Resuscitation. 1991; 21:57-60. [PubMed: 1852065]

22. Di Giantomasso D, May CN, Bellomo R. Norepinephrine and vital organ blood flow. Intensive Care Med. 2002; 28(12):1804-9. [PubMed: 12447527]

23. Sennoun N, Montemont C, Gibot S, Lacolley P, Levy B. Comparative effects of early versus delayed use of norepinephrine in resuscitated endotoxic shock. Crit Care Med. 2007; 35(7):173640. [PubMed: 17522580]

24. Mills LC, Moyer JH, Handley CA. Effects of various sympathicomimetic drugs on renal hemodynamics in normotensive and hypotensive dogs. Am J Physiol. 1960; 198:1279-83. [PubMed: 14422658]

25. Moss M, Burnham EL. Chronic alcohol abuse, acute respiratory distress syndrome, and multiple organ dysfunction. Critical Care Med. 2003; 31(4 Suppl)

26. Rivara FP, Jurkovich GJ, Gurney JG, Seguin D, Fligner CL, Ries R, Raisys VA, Copass M. Arch Surg. 1993; 128(8):907-12. discussion 912-3. [PubMed: 8102049]

27. Moss M, Parsons PE, Steinberg KP, Hudson LD, Guidot DM, Burnham EL, Eaton S, Cotsonis GA. Chronic alcohol abuse is associated with an increased incidence of acute respiratory distress syndrome and severity of multiple organ dysfunction in patients with septic shock. Crit Care Med. 2003; 31(3):869-77. [PubMed: 12626999]

28. Ono M, Yu B, Hardison EG, Mastrangelo MA, Tweardy DJ. Increased susceptibility to liver injury after hemorrhagic shock in rats chronically fed ethanol: role of nuclear factor-kappa B, interleukin-6, and granulocyte colony-stimulating factor. Shock. 2004; 21(6):519-25. [PubMed: 15167680]

29. Kuebler JF, Toth B, Yokoyama Y, Bland KI, Rue LW 3rd, Chaudry IH. Alpha1-acid-glycoprotein protects against trauma-hemorrhagic shock. J Surg Res. 2004; 119(1):21-8. [PubMed: 15126077]

30. Zentella de Piña M, Saldaña-Balmori Y, Hernández-Tobías A, Piña E. Nonsteroidal antiinflammatory drugs lower ethanol-mediated liver increase in lipids and thiobarbituric acid reactive substances. Alcohol Clin Exp Res. 1993; 17(6):1228-32. [PubMed: 8116836]

31. Castrejón-Sosa M, Villalobos-Molina R, Guinzberg R, Piña E. Adrenaline (via alpha(1B)adrenoceptors) and ethanol stimulate $\mathrm{OH}^{*}$ radical production in isolated rat hepatocytes. Life Sci. 2002; 71(21):2469-74. [PubMed: 12270752]

32. Kramer GC. Hypertonic resuscitation: physiologic mechanisms and recommendations for trauma care. J Trauma. 2003; 54(5 Suppl):S89-99. [PubMed: 12768109]

33. Woolf PD, McDonald JV, Feliciano DV, Kelly MM, Nichols D, Cox C. The catecholamine response to multisystem trauma. Arch Surg. 1992; 127(8):899-903. [PubMed: 1642533] 


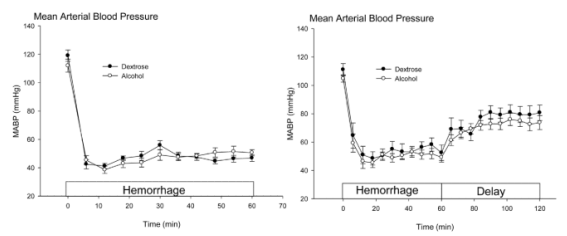

Figure 1.

Mean arterial blood pressure (MABP) in $\mathrm{mmHg}$ over time (min) during fixed-pressure hemorrhage in alcohol and dextrose-treated animals prior to immediate resuscitation $(\mathrm{n}=14$ 15) (left panel) and during hemorrhage and delay period prior to resuscitation $(n=12-13)$ (right panel). Values are mean \pm SEM. 

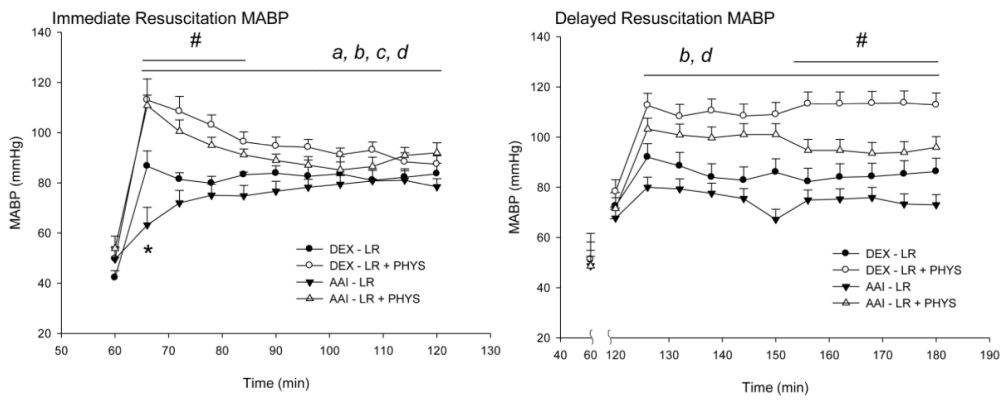

Figure 2.

Mean arterial blood pressure (MABP) in $\mathrm{mmHg}$ over time (min) during immediate $(\mathrm{n}=6-9)$ (left panel) and delayed $(\mathrm{n}=5-7)$ (right panel) fluid resuscitation \pm physostigmine $(100 \mu \mathrm{g} /$ $\mathrm{kg}$ ) of hemorrhaged animals. Values are mean \pm SEM. DEX: dextrose-treated; AAI: alcohol treated; LR: lactated Ringer's; PHYS: physostigmine. $a \mathrm{p}<0.05$ DEX-LR vs preresuscitation; $b \mathrm{p}<0.05$ DEX-LR+PHYS vs pre-resuscitation; $c \mathrm{p}<0.05$ AAI-LR vs preresuscitation; $d \mathrm{p}<0.05$ AAI-LR+PHYS vs pre-resuscitation; $* \mathrm{p}<0.05$ vs AAI-LR; \# $\mathrm{p}<0.05$ vs time- and treatment-matched LR-treated animals, by two-way repeated measures ANOVA. 

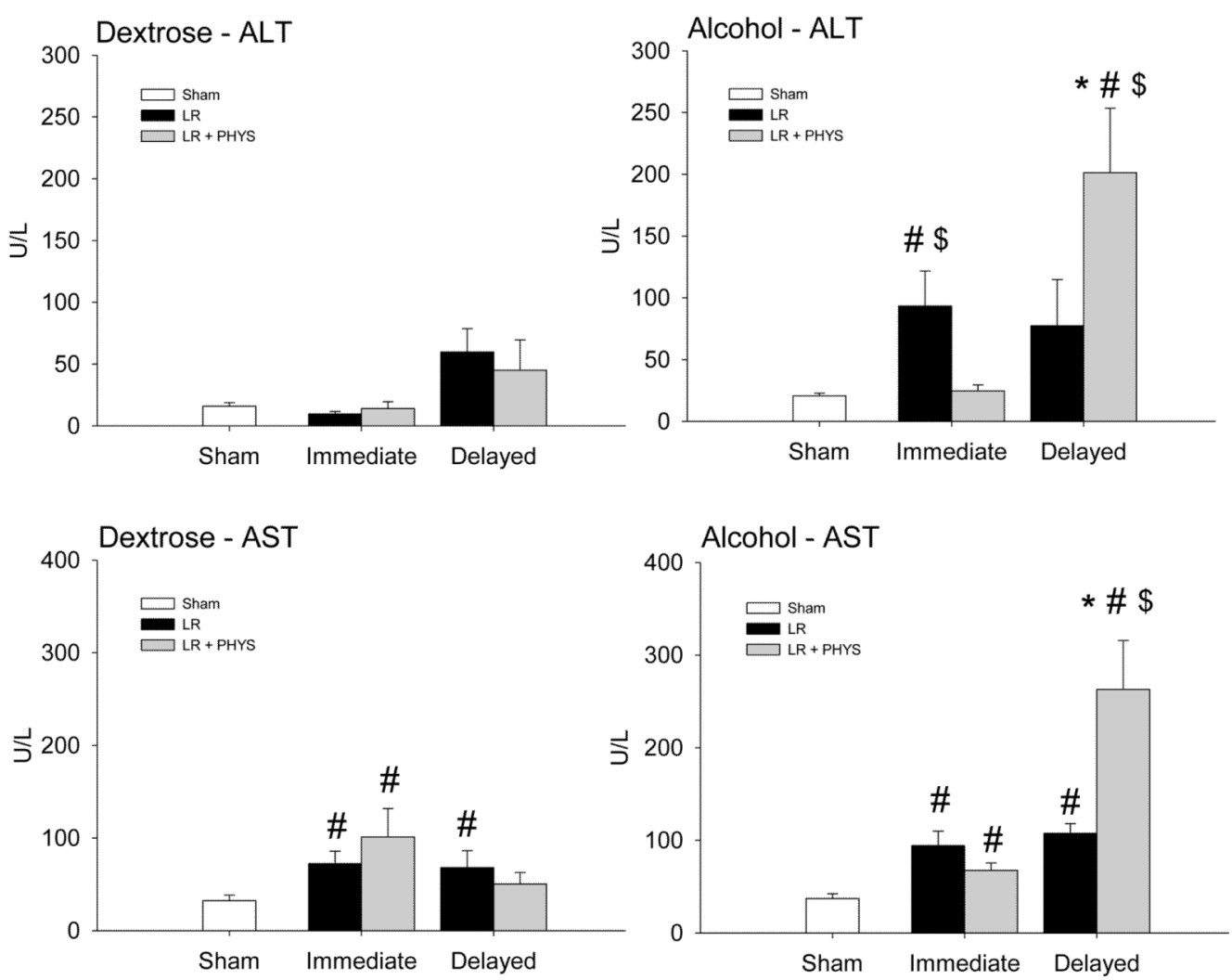

Figure 3.

Plasma levels of alanine aminotransferase (ALT) and aspartate aminotransferase (AST) measured at the completion of immediate $(n=6-9)$ and delayed $(n=5-7)$ fluid resuscitation with physostigmine $(100 \mu \mathrm{g} / \mathrm{kg})$ LR: lactated Ringer's; PHYS: physostigmine. Values are mean \pm SEM. \# $p<0.05$ vs time-matched non-hemorrhaged sham; * $p<0.05$ vs time-matched LR; $\$$ p $<0.05$ vs treatment-matched DEX, by two-way ANOVA. 

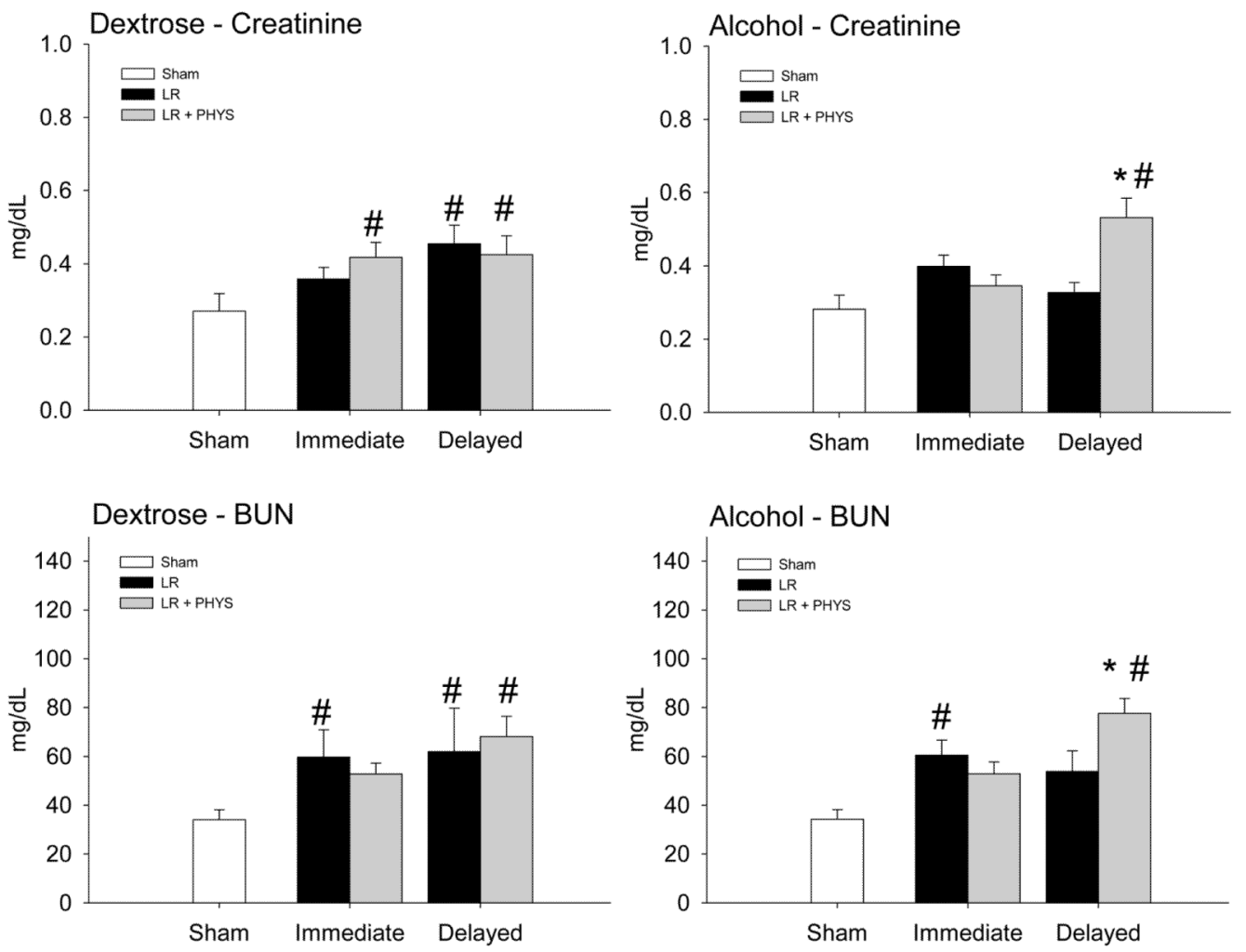

Figure 4.

Plasma levels of creatinine and blood urea nitrogen (BUN) measured at the completion of immediate $(\mathrm{n}=6-9)$ and delayed $(\mathrm{n}=5-7)$ fluid resuscitation with physostigmine $(100 \mu \mathrm{g} / \mathrm{kg})$; LR: lactated Ringer's; PHYS: physostigmine. Values are mean \pm SEM. \# p $<0.05$ vs timematched non-hemorrhaged sham; * $\mathrm{p}<0.05$ vs time-matched LR, by two-way ANOVA. 


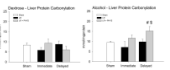

Figure 5.

Liver content of protein carbonyls measured one week post-hemorrhage following immediate $(n=4-6)$ and delayed $(n=6-7)$ fluid resuscitation with physostigmine $(100 \mu \mathrm{g} / \mathrm{kg})$. LR: lactated Ringer's; PHYS: physostigmine. Values are mean \pm SEM. \# $p<0.05$ vs timematched non-hemorrhaged sham; $\$ \mathrm{p}<0.05$ vs treatment-matched DEX, by two-way ANOVA. 